\title{
ON THE CONNECTION BETWEEN THE TOPOLOGICAL GENUS OF CERTAIN POLYHEDRA AND THE ALGEBRAIC GENUS OF THEIR HILTON-HOPF QUADRATIC FORMS
}

\author{
IMRE BOKOR
}

\begin{abstract}
The Hilton-Hopf quadratic form is defined for spaces of the homotopy type of a CW complex with one cell each in dimensions 0 and $4 n, K$ cells in dimension $2 n$ and no other cells. If two such spaces are of the same topological genus, then their Hilton-Hopf quadratic forms are of the same weak algebraic genus. For large classes of spaces, such as simply connected differentiable 4-manifolds, the converse is also true, as long as the suspensions of the spaces are also of the same topological genus. This note allays the conjecture that the converse is true in general by offering two techniques for generating infinite families of counterexamples.
\end{abstract}

It was shown in [1] that for $\mathcal{C}_{n}^{1}$, the class of those spaces which are of the same homotopy type as a $C W$-complex with precisely one cell in each of the dimensions $0,2 n$ and $4 n$, there is a Factorisation Theorem with respect to the one-point union (or V-product) of spaces. The theorem states that as long as the attaching map of the $4 n$-cell represents a homotopy class of infinite order in $\pi_{4 n-1}\left(S^{2 n}\right)$, the homotopy type of a finite wedge of such spaces determines the homotopy type of each of the spaces in the wedge. This stands in contrast to the situation where the attaching map is of finite order, for then non-cancellation phenomena occur with spaces of the same "genus", as shown in [3], [4], [5] and [6].

The proof of the Factorisation Theorem applied techniques which suggest a generalisation of the characterisation of the genus of a space in $\mathcal{C}_{n}^{1}$ to $\mathcal{C}_{n}^{K}$, the class of spaces of the homotopy type of a $C W$-complex with one cell in dimensions 0 and $4 n$ and a finite number $K$ of cells in dimension $2 n$. This larger class of spaces includes all $(2 n-1)$-connected differentiable $4 n$-manifolds. Up to homotopy, the spaces in $\mathcal{C}_{n}^{K}$ are mapping cones $C_{f}$ of continuous maps

$$
f: S^{4 n-1} \longrightarrow \bigvee_{k=1}^{K} S^{2 n}
$$


and hence are classified by $\pi_{4 n-1}\left(\bigvee_{k=1}^{k} S^{2 n}\right)$.

We fix $K$ and abbreviate $\bigvee_{k=1}^{K} S^{2 n}$ to $\bigvee S^{2 n}$.

For maps $f, g: S^{4 n-1} \rightarrow \bigvee S^{2 n}$ the set of homotopy classes of continuous maps between their mapping cones $C_{f}$ and $C_{g}$ is in bijection with the set of homotopy classes of homotopy commutative diagrams

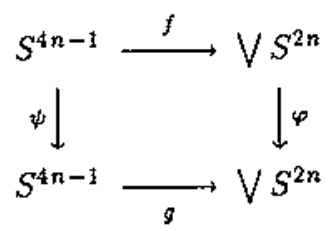

The set of homotopy classes of maps $\bigvee S^{2 n} \rightarrow \bigvee S^{2 n}$ admits a ring structure isomorphic to $M(K ; \mathbf{Z})$, the ring of $K \times K$ integral matrices. The function assigning to each self-map $\varphi$ of $S^{2 n}$ its degree induces one such isomorphism, by mapping $\varphi$ to the matrix $\underline{A}(\varphi)$ whose $(i, j)$-th coefficient is the degree of the composite map

$$
q_{i} \circ \varphi \circ i_{j}: S^{2 n} \longrightarrow \bigvee S^{2 n} \longrightarrow \bigvee S^{2 n} \longrightarrow S^{2 n},
$$

where

$$
i n_{j}: S^{2 n} \longrightarrow \bigvee S^{2 n}
$$

is the $j$-th canonical inclusion in the co-product and

$$
q_{i}: \bigvee S^{2 n} \longrightarrow S^{2 n}
$$

collapses each summand to the base point except the $i$-th one, on which it acts as the identity map.

It was shown in [1] that each homotopy class $[f] \in \pi_{4 n-1}\left(\bigvee S^{2 n}\right)$ can be represented by a pair consisting of the Hilton - Hopf quadratic form of $f$ and the suspension of $f$. The Hilton-Hopf quadratic form can itself be represented as a symmetric integral $K \times K$ matrix, $\underline{H}(f)$, whose entries are precisely the HittonHopf invariants of $f$, and the suspension by $\Sigma f$, a "column torsion vector" each of whose components is an element $\pi_{4 n}\left(S^{2 n+1}\right)$.

A suitable choice of generators for $\pi_{4 n-1}\left(V S^{2 n}\right)$ provides a simple way of computing $\varphi \circ f$ from $\varphi$ and $f$ by means of a matrix calculus:

$$
\underline{H}(\varphi \circ f)=\underline{A}(\varphi) \underline{H}(f)(\underline{A}(\varphi))^{t}
$$

and

$$
\Sigma(\varphi \circ f)=\underline{A}(\varphi) \Sigma(f) .
$$


Thus $\underline{H}(f)$ is a quadratic form which is an invariant of oriented homotopy type and the assignment to each $f \in \pi_{4 n-1}\left(V S^{2 n}\right)$ of its Hilton-Hopf quadratic form - that is, the quadratic form with matrix $\underline{H}(f)$ in the appropriate basis is natural in self-maps of $\bigvee S^{2 n}$. (Recall that the Hilton-Hopf quadratic form can also be thought of as the intersection form in the integral cohomology of $C_{f}$ )

The genus of a nilpotent space - more precisely, the genus of the homotopy type of a $C W$-complex of finite type - is defined to be the set of homotopy types of those nilpotent spaces, cach of whose $p$-localisations is homotopy equivalent to the $p$-localisation of the original space. Since we are only concerned with mapping cones $C_{f}$ of maps $f: S^{4 n-1} \rightarrow \bigvee S^{2 n}$, it follows from Lemma 2.2 of [1] that the two spaces $C_{f}$ and $C_{g}$ are of the same genus if and only if for each prime $p$ there is a homotopy commutative diagram

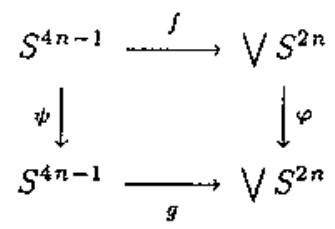

with both the degree of $\psi$ and the determinant of the matrix of $\varphi$ coprime to $p$. In terms of the matrix notation developed, this diagram is equivalent to equations

$$
\operatorname{deg}(\psi) \underline{H}(g)=\underline{A}(\varphi) \underline{H}(f)(\underline{A}(\varphi))^{l}
$$

and

$$
\operatorname{deg}(\psi) \sigma g=\underline{A}(\varphi) \sigma f
$$

with $\operatorname{deg}(\psi)$ and $\operatorname{det} \underline{A}(\varphi)$ coprime to $p$, where $\operatorname{det} A$ is the determinant of the matrix $A$.

Since the assignment of this quadratic form is natural, each of its invariants is, in fact, an invariant of the mapping cone of $f$. One such invariant is the genus of a quadratic form. An inmediate question is: What is the connection between the topological genus of the mapping cone of $f$ and the algebraic genus of its Hilton-Hopf quadratic form $\underline{H}(f)$ ?

The present paper is devoted to a study of this question, using the notation of [1].

The notion of genus in the theory of integral quadratic forms has a similar definition, namely two integral quadratic forms $\Phi$ and $\Phi^{\prime}$ of dimension $K$ are of the same genus precisely when for every prime $p$ they are equivalent over $Z_{p}$, the ring of $p$-adic integers. We recall the definition of equivalence of two quadratic forms over a ring in its more general form.

Definition. Let $\Lambda$ be a commutative ring of characteristic 0 , so that $Z$ is a subring of $\Lambda$. A $\Lambda$-quadratic form of dimension $K$ is a function $\Phi: M \rightarrow \Lambda$ from the free $\Lambda$-module $M$ of rank $K$ to $\Lambda$ satisfying $\Phi(\lambda x)=\lambda^{2} \Phi(x)$ for every 
$\lambda \in \Lambda$ and $x \in M$. The two $\Lambda$-quadratic forms $\Phi: M \rightarrow \Lambda$ and $\Phi^{\prime}: M^{\prime} \rightarrow \Lambda$ of dimension $K$ are said to be $\Lambda$-equivalent or equivalent over $\Lambda$ if there is an isomorphism $\tau: M \rightarrow M^{\prime}$ such that $\Phi=\Phi^{\prime} \circ \tau$.

Observe that any integral quadratic form may be considered to be a $\Lambda$. quadratic form. Hence it makes sense to speak of $\Lambda$-equivalence of integral quadratic forms. The definition of the genus of an integral quadratic form concerns the cases $\Lambda=Z_{p}$, the ring of $p$-adic integers, $p$ a prime number.

We transiate this definition into the language of matrices, since we shall be computing with matrices. Two integral quadratic forms represented by symmetric integral matrices $H$ and $H^{\prime}$ are of the same genus if and only if for each prime $p$ there is an invertible $p$-adic integral matrix $A$ such that

$$
H=A H^{\prime} A^{t} \text {. }
$$

Observe that this algebraic notion of genus is defined in terms of completion whereas the topological one was defined in terms of localisation. This difference disappears if only non-singular quadratic forms are considered, for then the ring $Z_{p}$ of $p$-adic integers may be replaced by $\mathbf{Z}_{(p)}$ the ring of $p$-local integers in the definition of the genus of an integral quadratic form ( $c f$. [2]). (Recall that $\mathbf{Z}_{\langle p\rangle}$ denotes the ring of $p$-local integers, that is the ring of all rational numbers with denominator in reduced form coprime to $p$.) In this case the similarities between the two concepts of genus are even more suggestive. Weakening the algebraic notion of genus slightly makes the connection clearer.

Deffnition. Let $\Lambda$ a commutative ring of characteristic 0 . Let two integral quadratic forms be given, one with the matrix $G$, the other with the matrix $H$ in some basis. Then the two forms are said to be weakly $\Lambda$-equivalent if there are an invertible $\Lambda$-matrix $A$ and $m$, a unit in $\Lambda$, with $m G=A H A^{t}$. (This condition is obviously independent of the choice of basis.) If $\Lambda=\mathrm{Z}_{(p)}$, then we speak simply of weak p-equivalence. Two non-singular integral quadratic forms are of the same weak genus if they are weakly $\mathbf{Z}_{\{p)^{-}}$-equivalent for every prime $p$.

It is an immediate consequence of the definitions that the weak genus of an integral quadratic form is an invariant of its genus.

Before examining examples of quadratic forms of the same weak genus, it should be observed that we may, without loss of generality, restrict ourselves to integral computations, for it is possible to "multiply up", as the next lemma makes clear.

Lemma 1. The integral quadratic forms $G$ and $H$ are $\mathbf{Z}_{(p)}$-equivalent if and only if there are an integral matrix $A$ and an integer $m$ with both $m$ and $\operatorname{det}(A)$ coprime to $p$, such that

$$
m G=A H A^{t}
$$

Proof: If $m^{\prime}$ is a unit in $\mathbf{Z}_{(p)}$ and if $A^{\prime}$ is an invertible $\mathbf{Z}_{(p)}$-matrix such that $m^{\prime} G=A^{\prime} H\left(A^{\prime}\right)^{t}$, then let $k$ be the least common multiple of the denominators 
of the entries in $A^{\prime}$ and the denominator of $m^{\prime}$. This $k$ is certainly a unit in $\mathbf{Z}_{(p)}$, as is the integer $m:=k^{2} m^{\prime}$. Moreover $A:=k A^{\prime}$ is an integral matrix invertible over $\mathbf{Z}_{(p)}$, so that its determinant (an integer) is a unit in $\mathbf{Z}_{(p)}$. But an integer is invertible in $\mathbf{Z}_{(p)}$ if and only if it is coprime to $p$. Finally, observe that

$$
m G=k^{2} m^{t} G=k A^{\prime} H k\left(A^{\prime}\right)^{t}=A H A^{t} .
$$

The weak genus of a quadratic form is frequently non-trivial. In fact the diagonal binary quadratic forms with a pair of distinct primes on the diagonal are always of the same weak genus, sometimes (but not always) of the same genus, but never equivalent, as the next results show.

Theorem 2. For each pair of distinct prime numbers $p$ and $q$, the integral quadratic forms with matrices

$$
\left(\begin{array}{ll}
p & 0 \\
0 & q
\end{array}\right) \text { and }\left(\begin{array}{cc}
1 & 0 \\
0 & p q
\end{array}\right)
$$

are of the same weak genus, but not equivalent.

Proof: Since

$$
p\left(\begin{array}{ll}
p & 0 \\
0 & q
\end{array}\right)=\left(\begin{array}{ll}
p & 0 \\
0 & 1
\end{array}\right)\left(\begin{array}{cc}
1 & 0 \\
0 & p q
\end{array}\right)\left(\begin{array}{ll}
p & 0 \\
0 & 1
\end{array}\right)
$$

the two forms are weakly $\mathbf{Z}_{(r)}$-equivalent for every prime $r \neq p$. But

$$
q\left(\begin{array}{ll}
p & 0 \\
0 & q
\end{array}\right)=\left(\begin{array}{cc}
0 & -1 \\
q & 0
\end{array}\right)\left(\begin{array}{cc}
1 & 0 \\
0 & p q
\end{array}\right)\left(\begin{array}{cc}
0 & q \\
-1 & 0
\end{array}\right)
$$

so that the two forms are weakly $\mathbf{Z}_{(p)}$-equivalent as well. The two forms

$$
\left(\begin{array}{ll}
p & 0 \\
0 & q
\end{array}\right) \text { and }\left(\begin{array}{cc}
1 & 0 \\
0 & p q
\end{array}\right)
$$

are equivalent oniy if the integral matrix equation

$$
\left(\begin{array}{cc}
1 & 0 \\
0 & p q
\end{array}\right)=\left(\begin{array}{ll}
a & b \\
c & d
\end{array}\right)\left(\begin{array}{ll}
p & 0 \\
0 & q
\end{array}\right)\left(\begin{array}{ll}
a & c \\
b & d
\end{array}\right)
$$

has a solution. This is only the case if the equation

$$
p a^{2}+q b^{2}=1
$$

has integral solutions, which it clearly never does. 
Lemma 3. The two quadratic forms

$$
\left(\begin{array}{cc}
2 & 0 \\
0 & 41
\end{array}\right) \text { and }\left(\begin{array}{cc}
1 & 0 \\
0 & 82
\end{array}\right)
$$

ate of the same genus.

Proof: The equality

$$
\left(\begin{array}{cc}
2 & 0 \\
0 & 41
\end{array}\right)=\left(\begin{array}{cc}
\frac{4}{7} & -\frac{1}{7} \\
\frac{41}{7} & \frac{2}{7}
\end{array}\right)\left(\begin{array}{cc}
1 & 0 \\
0 & 82
\end{array}\right)\left(\begin{array}{cc}
\frac{0}{7} & \frac{41}{7} \\
-\frac{1}{7} & \frac{2}{7}
\end{array}\right)
$$

shows that the two forms are $Z_{(p)}$-equivalent for $p \neq 7$ and the equality

$$
\left(\begin{array}{cc}
2 & 0 \\
0 & 41
\end{array}\right)=\left(\begin{array}{cc}
\frac{8}{109} & -\frac{17}{109} \\
\frac{697}{109} & \frac{4}{109}
\end{array}\right)\left(\begin{array}{cc}
1 & 0 \\
0 & 82
\end{array}\right)\left(\begin{array}{cc}
\frac{8}{109} & \frac{697}{109} \\
-\frac{17}{109} & \frac{4}{109}
\end{array}\right)
$$

shows that the two forms are $\mathbf{Z}_{(7)}$-equivalent.

Lermma 4. The two quadratic forms

$$
\left(\begin{array}{ll}
3 & 0 \\
0 & 5
\end{array}\right) \text { and }\left(\begin{array}{cc}
1 & 0 \\
0 & 15
\end{array}\right)
$$

are not of the same genus.

Proof: Let $k$ be an integer and $A$ the $2 \times 2$ integral matrix

$$
\left(\begin{array}{ll}
a & b \\
c & d
\end{array}\right)
$$

Then from the matrix equation

$$
k^{2}\left(\begin{array}{cc}
1 & 0 \\
0 & 15
\end{array}\right)=\left(\begin{array}{ll}
a & b \\
c & d
\end{array}\right)\left(\begin{array}{ll}
3 & 0 \\
0 & 5
\end{array}\right)\left(\begin{array}{ll}
a & c \\
b & d
\end{array}\right)
$$

it follows that

$$
k^{2}=3 a^{2}+5 b^{2}
$$

so that

$$
k^{2} \equiv 3 a^{2} \quad(\bmod 5)
$$

But for any integer $x$

$$
x^{2} \equiv 0, \pm 1 \quad(\bmod 5)
$$

so that $k$ must be divisible by 5 . In other words

$$
\left(\begin{array}{ll}
3 & 0 \\
0 & 5
\end{array}\right) \text { and }\left(\begin{array}{cc}
1 & 0 \\
0 & 15
\end{array}\right)
$$


are not 5-equivalent.

Turning to the connection between the topological genus of the mapping cone $C$ f of $f: S^{4 n-1} \rightarrow \bigvee S^{2 n}$ and the algebraic genus of $\underline{H}(f)$, the Hilton-Hopf quadratic form of $f$, the homotopy commutative diagram

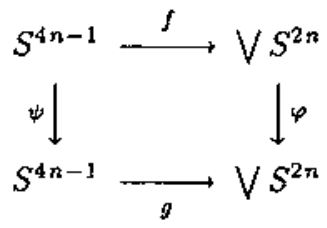

induces the algebraic equation

$$
\operatorname{deg}(\psi) \underline{H}(g)=\underline{A}(\varphi) \underline{H}(f) \underline{A}(\varphi)^{t}
$$

and $\psi$ and $\varphi$ are $p$-equivalences if and only if both $\operatorname{deg}(\psi)$ and $\operatorname{det}(\underline{A}(\varphi))$ are coprime to $p$ - in other words, if and only if $\underline{H}(f)$ and $\underline{H}(g)$ are weakly $p$ equivalent. The next theorem summarises these considerations.

Theorem 5. The mapping cones $C_{f}$ and $C_{g}$ are of the same genus only if $\underline{H}(f)$ and $\underline{H}(g)$ are of the same weak genus.

Thus the weak genus of the Hilton-Hopf quadratic form $\underline{H}(f)$ is an invariant of the genus of $C_{f}$, as is the genus of the suspension of $C_{f}$. In fact, these two invariants characterise the genus of $C_{f}$ in the case that the bouquet of spheres $V S^{2 n}$ consists of precisely one sphere, for in that case the Hilton-Hopf quadratic form reduces to the classical Hopf invariant of $f$ which is a single integer; and two integers - integral quadratic forms of dimension 1 - are of the same weak genus if and only if they agree up to sign. Hence the Classification Theorem of [1] can be reformulated as

Theorem 6. Two spaces in $\mathcal{C}_{n}^{1}$ are of the same genus if and only if

(i) their Hilton-Hopf quadratic forms are of the same weak genus, and

(ii) their suspensions are of the same genus.

This reformulation invites the conjecture that the result generalises to the case in which the bouquet contains an arbitrary (finite) number of $2 n$-spheres.

Conjecture. Two spaces in $\mathcal{C}_{n}^{K}$ are of the same genus if and only if

(i) their Hilton-Hopf quadratic forms are of the same weak genus, and

(ii) their suspensions are of the same genus.

Of course the "only if" part of the conjecture is certainly true. 
For $K=0$ the conjecture is true because any space in $\mathcal{C}_{n}^{0}$ is homotopy equivalent to $S^{4 \pi}$, being a simply connected Moore space with the appropriate homology. Theorem 6 asserts the conjecture to be true for $K=1$. The conjecture is also clearly true if the torsion subgroup of $\pi_{4 n-1}\left(S^{2 n}\right)$ is trivial, for then the equation involving the torsion components imposes no additional constraint, and every $K \times K$ integral matrix can be realised as a self-map of $V S^{m}$, for any $m>1$.

Nevertheless, if $K \geq 2$ and if the torsion subgroup of $\pi_{4 n-1}\left(S^{2 n}\right)$ is nontrivial, then counterexamples to the conjecture can be systematically constructed. Two schemata follow for doing so for $n \notin\{1,2,4\}$ when the kernel of the suspension map $\Sigma: \pi_{4 n-1}\left(\bigvee S^{2 n}\right) \rightarrow \pi_{4 n}\left(V S^{2 n+1}\right)$ consists precisely of those classes $[f]$ whose torsion component is trivial.

Counterexample 1. If the torsion subgroup of $\pi_{4 n-1}\left(S^{2 n}\right)$ is non-trivial, then there are mapping cones of maps $S^{4 n-1} \rightarrow S^{2 n} \vee S^{2 n}$ with the following properties.

(i) Their Hilton-Hopf quadratic forms are of the same weak genus.

(ii) Their suspensions are homotopy equivalent.

(iii) They are not themselves of the same genus.

Counterexample 2. If the torsion subgroup of $\pi_{4 n-1}\left(S^{2 n}\right)$ is not cyclic, then there are mapping cones of maps $S^{4 n-1} \rightarrow S^{2 n} \vee S^{2 n}$ with the following properties.

(i) Their Hilton-Hopf quadratic forms are equivalent.

(ii) Their suspensions are homotopy equivalent.

(iii) They are not themselves of the same genus.

Construction of Counterexample 1. We assume that $T$, the torsion subgroup of $\pi_{4 n-1}\left(S^{2 n}\right)$, is non-trivial. Let $p$ be a prime divisor of $t$, the order of $T$. Then $T$ has an element $x$ of order $p$. Let $q$ be any prime number other than $p$. Finally choose $f, g: S^{4 n-1} \rightarrow S^{2 n} \vee S^{2 n}$ with the following properties:

$$
\begin{aligned}
& \underline{H}(f)=\left(\begin{array}{cc}
2 p & 0 \\
0 & 2 q
\end{array}\right) \text { and } \Sigma(f)=\left(\begin{array}{l}
x \\
0
\end{array}\right) \\
& \underline{H}(g)=\left(\begin{array}{cc}
2 & 0 \\
0 & 2 p q
\end{array}\right) \text { and } \Sigma(g)=\left(\begin{array}{l}
x \\
0
\end{array}\right)
\end{aligned}
$$

Then Theorem 2 asserts that $\underline{H}(f)$ and $\underline{H}(g)$ are of the same weak genus, verifying (i).

The suspensions are clearly homotopically equivalent - in fact both are homotopically equivalent to $C_{\Sigma x} \vee S^{2 n+1}$ - which establishes (ii).

Now consider the homotopy commutative diagram

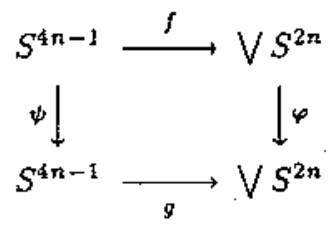


where $\psi$ has degree $m$ and $\underline{A}(\varphi)=\left(\begin{array}{ll}a & b \\ c & d\end{array}\right)$

It follows from the equation

$$
m \underline{H}(g)=\underline{A}(\varphi) \underline{H}(f)(\underline{A}(\varphi))^{t}
$$

that

$$
m p q=p c^{2}+q d^{2}
$$

so that $p$ divides $d$. Moreover, the equation

$$
\underline{A}(\varphi) \Sigma f=m \Sigma g
$$

shows that $c x=0$, so that $p$, which is the order of $x$, also divides $c$. But then $p$ divides $\operatorname{det}(\underline{A}(\varphi))$ as well, so that for no such diagram is $\varphi$ a $p$-equivalence. This establishes (iii) thereby establishing the first family of counterexamples to the conjecture.

Construction of Counterexample 2. For the second construction we consider the case when the torsion subgropup $T$ of $\pi_{4 n-1}\left(S^{2 n}\right)$ is not cyclic. In that case it has a subgroup $G$ isomorphic to $\mathbf{Z} / p \mathbf{Z} \oplus \mathbf{Z} / p \mathbf{Z}$ for some prime $p$ We identify $G$ with $\mathbf{Z} / p \mathbf{Z} \oplus \mathbf{Z} / p \mathbf{Z}$.

Choose an integer $r$ with $0<r<p$. Then there are integers $s$ and $t$ with $r t-p s=-1$. We may assume without any loss of generality that $0<t<p$, for $r(t+k p)-s p=r t-(s-r k) p$. From this it also follows that $0<s<p$. Put

$$
\zeta:=\left(\begin{array}{cc}
r & p \\
s & t
\end{array}\right)
$$

Then clearly $\zeta \in \mathrm{G} L(2 ; \mathbf{Z})$, with inverse

$$
\zeta^{-1}=\left(\begin{array}{cc}
-t & p \\
s & -r
\end{array}\right)
$$

Writing the elements of $G$ as rows means that the rows of $\zeta^{-1}$ can be viewed as elements of $G$, and hence taken to represent homotopy classes of maps $S^{4 n-1} \rightarrow$ $S^{2 n}$. The first row corresponds to the element $(-t, 0)$ of $G$ and the second to $(s,-r)$. If, furthermore, the elements of $G^{2}$ are written as columns with elements of $G$ as entries, then we may regard $\zeta^{-1}$ as an element $\sigma$ of $G^{2}$ and so as representing a homotopy class of maps $S^{4 n-1} \rightarrow S^{2 n} \vee S^{2 n}$. Put $y:=\zeta \sigma$. Then $y$ is the $2 \times 2$ unit matrix 1 viewed as an element of $G^{2}$ and $\zeta$ defines an automorphism of $G^{2}$ mapping $x$ to $y$. Now choose $f, g: S^{4 n-1} \rightarrow S^{2 n} \vee S^{2 n}$ with the following properties

$$
\begin{aligned}
& \underline{H}(f)=\left(\begin{array}{ll}
2 & 0 \\
0 & 2
\end{array}\right) \text { and } \Sigma(f)=\left(\begin{array}{cc}
-t & p \\
s & -r
\end{array}\right) \\
& \underline{H}(g)=\left(\begin{array}{ll}
2 & 0 \\
0 & 2
\end{array}\right) \text { and } \Sigma(g)=\left(\begin{array}{ll}
1 & 0 \\
0 & 1
\end{array}\right)
\end{aligned}
$$


The Hilton-Hopf quadratic forms $\underline{H}(f)$ and $\underline{H}(g)$ are identical.

Equally, the suspensions $\Sigma C_{f}$ and $\Sigma C_{g}$ are homotopy equivalent. For example, any self-map $\varphi$ of $S^{2 n+1} \vee S^{2 n+1}$ with $\underline{A}(\varphi)=\zeta$ together with the identity map of $S^{4 n}$, defines a suitable homotopy equivalence from $\Sigma C_{f}$ to $\Sigma C_{g}$.

Now consider the homotopy commutative diagram

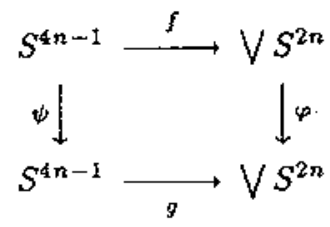

where $\psi$ has degree $m$ and $\underline{A}(\varphi)=\left(\begin{array}{ll}a & b \\ c & d\end{array}\right)$

It follows from

$$
m \underline{H}(g)=\underline{A}(\varphi) \underline{H}(f)(\underline{A}(\varphi))^{t}
$$

that

$$
\underline{A}(\varphi)(\underline{A}(\varphi))^{t}=m \underline{1}
$$

so that

$$
\varphi=\left(\begin{array}{cc}
a & b \\
-\lambda b & \lambda a
\end{array}\right)
$$

with $\lambda^{2}=1$.

A further direct computation using $\psi \underline{y}=\varphi \underline{x}$ shows that

$$
a p-r b \equiv \lambda(b t+a s) \equiv 0(\bmod p),
$$

from which it follows successively that $p$ divides both $b$ and $a$, since $r, s$ and $\lambda$ are all coprime to $p$. Thus $\varphi$ cannot be a $p$-equivalence, which establishes the construction for the second family of counterexamples.

Remarks. These counterexamples also dispose of a number of possible refinements of the conjecture, for the spaces constructed satisfy more stringent requirements than set out in the conjecture. The second recipe provides a method for constructing couriterexamples with the Hilton-Hopf forms actually coinciding and the suspensions being homotopically equivalent. All that is needed for this is that the torsion subgroup of $\pi_{4 n-1}\left(S^{2 n}\right)$ not be cyclic, as is the case, for example, for $n=5,8,9$. It should however be noted that in none of the counterexamples constructed does the Hilton-Hopf quadratic form have determinant 1 , so that none of the spaces is a manifold.

It remains to be seen whether the conjecture be true for manifolds and also to find some invariant, additional to (i) and (ii) of the discredited conjecture, which will guarantee that $C_{f}$ and $C_{g}$ are of the same genus. 


\section{References}

1. BoKor, I., "Genus and cancellation in homotopy theory," (to be published).

2. CaSsels, J.W., "Rational quadratic forms," London, Academic Press, 1978.

3. FreYd, P., Stable homotopy II, AMS Proc. of Symp. in Pure Math. 18 (1970), 161-183.

4. Hilton, P.J., The grothendiek group of compact poiyhedra, Fundamenta Mathematice 61 (1967), 199-214.

5. Molnar, E., Relation between wedge cancellation and localization for complexes with two cells, J. of P. and Appl. Alg. 3 (1972), 77-81.

6. Wilkerson, C., Genus and cancellation, Topology 14 (1975), 29-36.

75 Nount St

Coogee 2034

AUSTRALIA

Rebut el 17 de Desembre de 1989 